Published in final edited form as:

Nat Chem Biol. 2008 March ; 4(3): 145-147. doi:10.1038/nchembio0308-145.

\title{
Assembling the pieces
}

\author{
Dennis J Thiele and \\ Department of Pharmacology and Cancer Biology, Duke University School of Medicine, Research \\ Drive, LSRC C351, Durham, North Carolina 27710, USA \\ Jonathan D Gitlin \\ Edward Mallinckrodt Department of Pediatrics, Washington University School of Medicine, 660 \\ South Euclid Avenue, Box 8208, St. Louis, Missouri 63110, USA
}

Dennis J Thiele: dennis.thiele@duke.edu; Jonathan D Gitlin: gitlin@wustl.edur

\section{Abstract}

Transition metals function as cofactors in specific proteins, catalyzing electron exchange reactions, binding substrates and stabilizing protein structure. Studies of human diseases and of model organisms have defined many of the details of metal uptake, trafficking, and excretion. The current challenge is to integrate these details into a view of metal content, speciation, localization and use within organisms and ecosystems.

Transition metals serve an essential role in the metabolism of all living organisms, and yet, unlike most metabolites, these inorganic elements are neither produced nor consumed during such reactions. For this reason, the study of transition metal metabolism resides at a unique interface of chemistry and biology. In the broadest perspective, the transition metals with essential functions in cellular biochemistry are required for nitrogen fixation, oxygenic photosynthesis and aerobic respiration and thus link all organisms through maintenance of the global electron exchange that sustains our biosphere ${ }^{1}$. The availability of these elements, and their unique chemistries, has therefore served as a driving force for the evolution of life on Earth, and, in this regard, transition metal metabolism is directly dependent upon the biogeochemical oceanic and atmospheric cycles of the planet ${ }^{2}$. For example, the rise of atmospheric oxygen accompanying photosynthesis dramatically influenced the availability of specific metals, and phylogenetic and proteomic analysis reveals clear evidence of metalloprotein evolution reflecting these ancient shifts in geochemistry ${ }^{3}$. Intriguingly, although adaptation to oxygen occurred independently in organisms of diverse lineage ${ }^{4}$, elucidation of the mechanisms of transition metal metabolism has revealed a striking evolutionary conservation among all lineages, a finding that underscores the importance of metal availability as a critical evolutionary factor. This concept is further supported by metagenomic and metabolic studies within unusual ecosystems that reveal the essential nature of transition metals in maintaining extremophile habitats that give rise to organismal diversity under a plethora of geochemical conditions ${ }^{5}$.

From this perspective, we can consider the systems biology of transition metal metabolism, where the metallome is defined as the cellular metal content, speciation (the identification and quantitation of specific forms of an element), and localization in each cell and $\operatorname{organism}^{6}$, dependent upon the dynamic inorganic geochemistry of the Earth and resulting in a specific metalloproteome that is the functional unit of transition metal biology. Notably, although geochemistry provides information about the environment and genomic analysis yields information about which RNA molecules and proteins may be present, a systematic view of transition metal metabolism requires an understanding of how organisms sense, adapt and use these metals within the biodiversity characteristic of each specific and dynamic ecosystem or biome, where the metallobiome defines the metallome of each 
ecosystem. An elegant example of this concept is methanotrophic bacteria, critical components of the global methane cycle that use copper-dependent methane monooxygenase to oxidize methane. Recent studies have revealed that methane monooxygenase expression in nature is largely controlled by solid-phase copper geochemistry and that the relative ability of metal acquisition systems in methanotrophs to obtain copper from these mineral sources is the primary determinant of methane oxidation patterns in the natural environment ${ }^{7}$. Although little is understood at present about such ecological considerations in human transition metal metabolism, they are likely to be of critical importance. For example, knowledge of the intestinal microbiome suggests that transition metals are required to support diverse ecosystems that in turn have a profound influence upon human physiology and metabolism ${ }^{8}$. In this latter context, recent observations of a critical role for copper homeostasis in fungal and bacterial pathogenesis underscore the importance of ecological contexts in host-pathogen interactions relevant to human disease $\mathrm{e}^{9,10}$.

In this transcendent view of transition metal metabolism, elucidation of the metallobiome reveals the language through which the genome and the environment communicate. This is a dynamic process, changing in response to what the environment offers, the specific needs of the organisms and the molecular details that are unique to and dependent upon the chemistry and biology of each element. Although insights into such processes are incipient, we provide here a perspective that may suggest avenues for future exploration.

\section{New imaging methodologies}

Recent advances in imaging have provided much needed insight into the dynamics of protein and membrane trafficking within cells and organelles and are essential for a dynamic view of proteome function. Similarly, real time, in vivo analysis of cellular metal content and trafficking will be required to elucidate a systematic view of metal metabolism. Previous approaches have involved combining genome-wide mutational analysis in Saccharomyces cerevisiae ${ }^{11}$ or in Arabidopsis thaliana roots ${ }^{12}$ with inductively coupled plasma-atomic emission spectroscopy to determine elemental profiles and define genes essential in metal metabolism. These studies estimate that up to 4\% of the Arabidopsis genome is involved in regulating the plant's nutrient and trace-element metabolism and suggest new mechanistic hypotheses regarding metal metabolism based upon identification of previously uncharacterized genes. More recent bioinformatics approaches have identified putative copper-binding proteins encoded in the genomes of fifty-seven organisms, from bacteria to archaea to eukaryotes, with the size of the copper proteome estimated to be about $1 \%$ of the total proteome of an organism ${ }^{13}$.

Although such approaches provide a useful first pass view of the genomics of transition metal metabolism, the lack of any dynamic information remains a significant limitation. For example, in populations with crop-based diets, iron deficiency affects more than 500 million children, and any effort to increase the iron content of staple foods will require this dynamic view of iron metabolism ${ }^{14}$. This is underscored by a recent series of experiments combining mutational analysis and imaging to demonstrate an essential role for iron in seedling development in Arabidopsis $^{15}$. Although abundant, iron is largely insoluble, and the challenge for all organisms is to acquire adequate amounts while avoiding toxicity ${ }^{16}$. Kim et al. identified VIT1 as the Arabidopsis ortholog of a vacuolar yeast iron importer, CCC1, that functions in iron compartmentalization and storage. Although VIT1 rescued the ironsensitive phenotype of a yeast mutant lacking CCC1 and was highly expressed in developing seeds, no difference was detected in the total iron content of seeds or shoots between wildtype plants and mutants that lack the VIT1 gene. However, X-ray fluorescence microtomography demonstrated a dramatic loss of iron in germinating seeds that lacked 
VIT1, specifically in provascular cells of the hypocotyl, radicle and cotyledon embryonic seed tissues (Fig. 1). These data implicate the vacuole of provascular cells as critical to iron storage in wild-type seeds, and, consistent with this, seedlings germinate poorly under conditions of limited soil iron availability. These findings demonstrate the importance of noninvasive, three-dimensional quantitative element analysis in living samples to our understanding of trace-metal metabolism and evaluation of the metallobiome in specific ecosystems.

$\mathrm{X}$-ray fluorescence microscopy can also provide information regarding metal speciation with a method of X-ray absorption spectroscopy referred to as micro-XANES spectroscopy ${ }^{17}$. Using this method in combination with a specific fluorescence sensor, Yang et al. provided clear evidence in support of a kinetically labile copper pool, with near-edge features characteristic for monovalent copper, distributed specifically in the mitochondria and late Golgi ${ }^{18}$. More recently, the application of X-ray fluorescence microscopy to tissue culture models of angiogenesis revealed dramatic relocalization of copper from intracellular compartments to the tips of endothelia during capillary growth and subsequent impairment of capillary network formation after copper chelation ${ }^{19}$. These studies underscore the value of in situ image analysis of transition metal metabolism in dynamic processes in which specific biological roles may be illuminated.

\section{New integrative model systems}

A systems biology approach to understanding transition metal metabolism will require the development of robust models that both are genetically tractable and permit broad, physiologic interrogation. An elegant example of such an approach is recent studies examining copper homeostasis during early development. Ctr1 is a homotrimeric protein, conserved from yeast to humans, that transports copper across the plasma membrane with high affinity and specificity ${ }^{20}$. Although the deletion of Ctr1 from the mouse germline is embryonic lethal, the generation of intestinal epithelial cell-specific Ctr1 knockout mice revealed striking neonatal defects in copper accumulation in peripheral tissues, hepatic iron overload, cardiac hypertrophy and severe growth and viability defects ${ }^{21}$. The growth and viability in these mice are rescued by postnatal parenteral copper administration, revealing a critical role for intestinal $\mathrm{Ctr} 1$ in neonatal copper homeostasis and identifying $\mathrm{Ctr} 1$ as the main factor driving intestinal copper absorption in mammals. Extension of these studies to Drosophila demonstrated that copper import by $\mathrm{Ctr} 1$ is required to drive neuropeptide maturation during normal larval growth and development ${ }^{22}$. The importance of such models wherein specific genetic interactions can be dissected in a physiologic context is underscored by recent biochemical observations that Ctr1 functions as a key regulator of the differentiation capacity of both stem and progenitor cell populations, independent of the copper transport functions ${ }^{23}$. Taken together these findings reveal the utility of such models in illuminating the specifics of transition metal metabolism in the context of the whole organism.

The metabolism of transition metals is carefully regulated because the chemical properties that render these metals useful are also responsible for toxicity. Regulation of these processes is dynamically responsive to supply and demand and is therefore multilayered in metazoans, where both developmental processes and cell-specific requirements add complexity to transition metal metabolism. Recent studies reveal the utility of zebrafish as a potential approach to such questions in vertebrates, because the optical clarity and rapid external development of these embryos permits detailed characterization of deficient phenotypes and both small-molecule and genetic screens permit the identification of specific phenotypes ${ }^{24,25}$. Using such a chemical genetic screen to find mutant embryos having a phenotype similar to that produced by copper deficiency resulted in identification of 
calamity, a mutant defective in the zebrafish ortholog (atp7a) of the Menkes disease gene ${ }^{26}$. The gene dosage of atp $7 a$ determines the sensitivity to copper deprivation, revealing a developmental hierarchy of copper metabolism that is informed by specific genetic factors (Fig. 2). These findings provide insight into the developmental pathophysiology of copper metabolism and suggest mechanisms whereby suboptimal copper metabolism may contribute to pathogenesis of structural birth defects of the axial skeleton ${ }^{27}$. These studies illustrate the valuable insights into the systems biology of transition metal metabolism provided by genetically tractable model organisms and suggest new avenues of exploration in this field.

\section{New integrated computational bioinformatic methodology}

Future studies in transition metal metabolism should provide insight into how environmental and genetic perturbations result in dramatic changes in organismal biology. Despite the application of several high-throughput technologies, including genome-wide association, metalloproteomics and transcriptomics, few such approaches have yet to offer such an accurate or integrated view of transition metal metabolism. Indeed, we would argue that such an understanding will require the development of robust computational bioinformatic methods that can be integrated with knowledge of genomics, metabolism and small molecule chemistry ${ }^{28}$. This concept is illustrated in a report demonstrating that under conditions of iron deficiency a dramatic genome-wide metabolic reprogramming occurs that invokes selective mRNA degradation ${ }^{29}$. DNA micro-array experiments demonstrated that mRNAs for $\sim 30$ genes, encoding proteins involved in iron uptake and iron-dependent sterol and fatty acid biosynthesis, are transcriptionally elevated in response to low iron availability. Surprisingly, the steady state levels of over $\sim 80$ more mRNAs were modestly to severely decreased under the same iron-limited conditions. Importantly, an integrated computational bioinformatic analysis indicated that this latter group is composed of genes involved in critical metabolic functions including the tri-carboxylic acid (Krebs) cycle, mitochondrial oxidative phosphorylation, biotin synthesis, heme and iron-sulfur cluster assembly and many other known or predicted processes that are involved in iron storage or metabolic reactions that are iron dependent ${ }^{29}$.

Importantly, the development of new computational bioinformatic approaches has begun to permit accurate prediction of broad organismal metabolic and environmental responses and could allow a reasonable view of functional biological networks for transition metal metabolism. Indeed, such an approach was recently used with a poorly defined archaean species to accurately define the genetic (transcriptional) adaptive responses to various environmental factors that included manganese, cobalt, iron, copper and $z i n c^{30}$. This extraordinarily effective systems biology approach highlights the essential need for predictive computational biology that can be integrated with high-throughput '-omic' technologies and reveals the power of such techniques to provide an organismal view of transition metal metabolism even in circumstances where biological habitat is poorly understood. The application of such approaches to model systems in which most of the molecular pieces of metal metabolism have been defined promises to yield exciting new knowledge in this field.

\section{Conclusions}

In the past decade, molecular genetic analyses of human diseases, as well as numerous studies in model organisms, have defined many of the molecules involved in the uptake, transport, trafficking, storage and excretion of iron, zinc and copper, the three transition metals predominant in human physiology ${ }^{31-33}$. Although this reductionist approach has been essential, the exciting challenge in this field now is to integrate these pieces into a 
systematic view of the dynamic metabolism of transition metals. These efforts will require broad input from such diverse fields as genomics, geochemistry, ecology, evolutionary biology, microbiology, proteomics and molecular biology and have the potential to reveal both the complex mechanisms of transition metal interaction and the influences of such processes on the origin of ecosystems and the driving forces of evolution.

The availability of new model systems, imaging technology and computational bioinformatics tools could now make such approaches possible, and they await the next generation of young investigators to drive this exciting field forward.

\section{Acknowledgments}

We thank members of our laboratories for insights and discussions on the ideas presented here. The work presented from the authors laboratories was supported by US National Institutes of Health grants DK44464 (J.D.G.), DK61763 (J.D.G.), HD39522 (J.D.G.), GM41840 (D.J.T.), DK074192 (D.J.T.) and 5P42ES010356 (D.J.T.).

\section{References}

1. Falkowski PG. Science. 2006; 311:1724-1725. [PubMed: 16556831]

2. Reysenbach AL, Shock E. Science. 2002; 296:1077-1082. [PubMed: 12004120]

3. Dupont CL, Yang S, Palenik B, Bourne PE. Proc. Natl. Acad. Sci. USA. 2006; 103:17822-17827. [PubMed: 17098870]

4. Raymond J, Segre D. Science. 2006; 311:1764-1767. [PubMed: 16556842]

5. Reysenbach AL, et al. Nature. 2006; 442:444-447. [PubMed: 16871216]

6. Williams RJP. Coord. Chem. Rev. 2001; 216:583-595.

7. Knapp CW, Fowle DA, Kulczycki E, Roberts JA, Graham DW. Proc. Natl. Acad. Sci. USA. 2007; 104:12040-12045. [PubMed: 17615240]

8. Ley RE, Peterson DA, Gordon JI. Cell. 2006; 124:837-848. [PubMed: 16497592]

9. Waterman SR, et al. J. Clin. Invest. 2007; 117:794-802. [PubMed: 17290306]

10. Liu T, et al. Nat. Chem. Biol. 2007; 3:60-68. [PubMed: 17143269]

11. Eide DJ, et al. Genome Biol. 2005; 6:R77. [PubMed: 16168084]

12. Lahner B, et al. Nat. Biotechnol. 2003; 21:1215-1221. [PubMed: 12949535]

13. Andreini C, Banci L, Bertini I, Rosato A. J. Proteome Res. 2007; 7:209-216. [PubMed: 17988086]

14. Gitlin JD. Science. 2006; 314:1252-1253. [PubMed: 17124312]

15. Kim SA, et al. Science. 2006; 314:1295-1298. [PubMed: 17082420]

16. Hentze MW, Muckenthaler MU, Andrews NC. Cell. 2004; 117:285-297. [PubMed: 15109490]

17. Fahrni CJ. Curr. Opin. Chem. Biol. 2007; 11:121-127. [PubMed: 17353139]

18. Yang L, et al. Proc. Natl. Acad. Sci. USA. 2005; 102:11179-11184. [PubMed: 16061820]

19. Finney L, et al. Proc. Natl. Acad. Sci. USA. 2007; 104:2247-2252. [PubMed: 17283338]

20. Nose Y, Rees EM, Thiele DJ. Trends Biochem. Sci. 2006; 31:604-607. [PubMed: 16982196]

21. Nose Y, Kim BE, Thiele DJ. Cell Metab. 2006; 4:235-244. [PubMed: 16950140]

22. Turski ML, Thiele DJ. J. Biol. Chem. 2007; 282:24017-24026. [PubMed: 17573340]

23. Haremaki T, Fraser ST, Kuo YM, Baron MH, Weinstein DC. Proc. Natl. Acad. Sci. USA. 2007; 104:12029-12034. [PubMed: 17620605]

24. Peterson RT, Link BA, Dowling JE, Schreiber SL. Proc. Natl. Acad. Sci. USA. 2000; 97:1296512969. [PubMed: 11087852]

25. Zon LI, Peterson RT. Nat. Rev. Drug Discov. 2005; 4:35-44. [PubMed: 15688071]

26. Mendelsohn BA, et al. Cell Metab. 2006; 4:155-162. [PubMed: 16890543]

27. Gansner JM, Mendelsohn BA, Hultman KA, Johnson SL, Gitlin JD. Dev. Biol. 2007; 307:202213. [PubMed: 17543297]

28. Oprea TI, Tropsha A, Faulon JL, Rintoul MD. Nat. Chem. Biol. 2007; 3:447-450. [PubMed: 17637771] 
29. Puig S, Askeland E, Thiele DJ. Cell. 2005; 120:99-110. [PubMed: 15652485]

30. Bonneau R, et al. Cell. 2007; 131:1354-1365. [PubMed: 18160043]

31. Rees EM, Thiele DJ. Curr. Opin. Microbiol. 2004; 7:175-184. [PubMed: 15063856]

32. Eide DJ. Biochim. Biophys. Acta. 2006; 1763:711-722. [PubMed: 16675045]

33. Andrews NC, Schmidt P. J. Annu. Rev. Physiol. 2007; 69:69-85. 

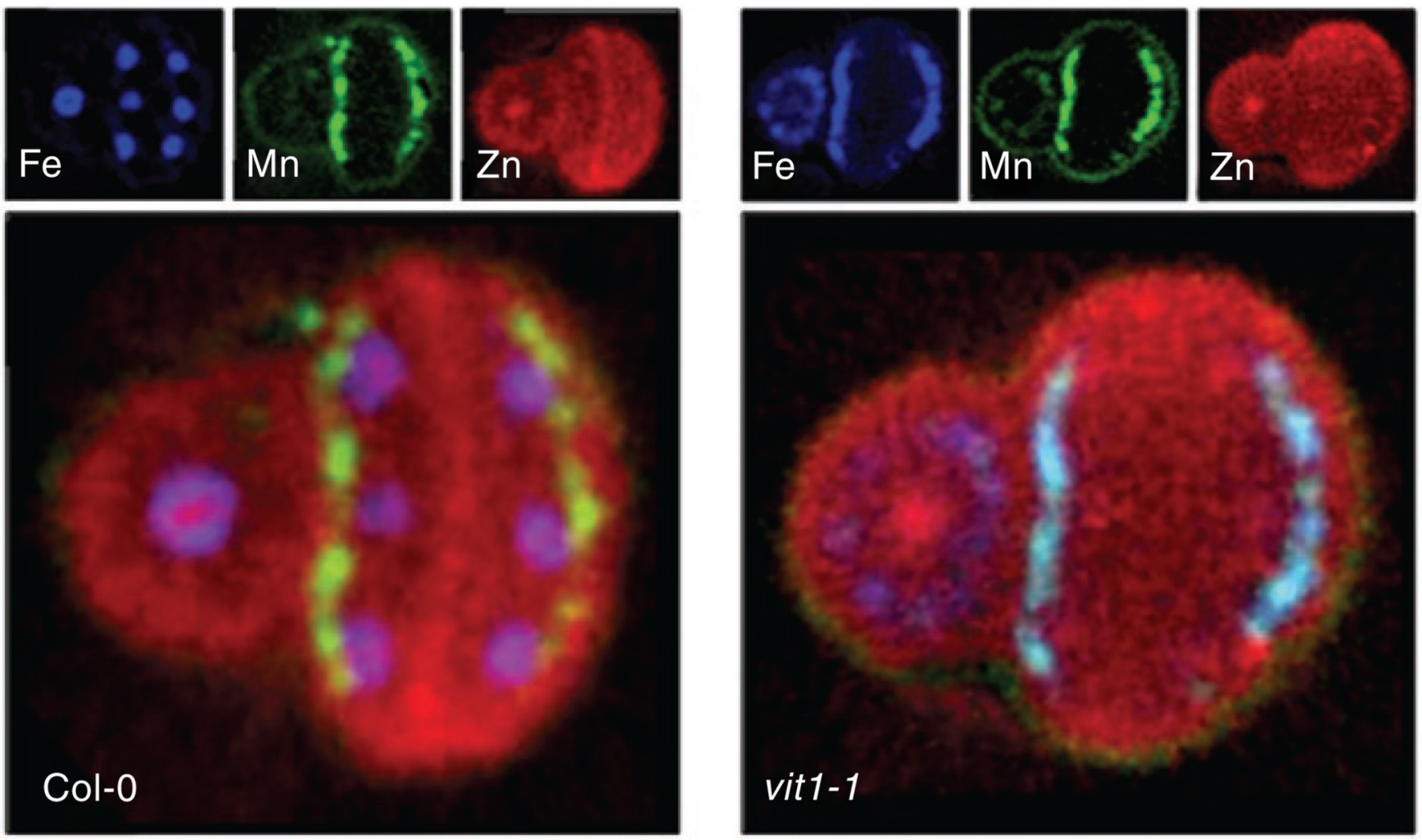

Figure 1.

X-ray fluorescence microtomography of Arabidopsis seed. X-ray fluorescence tomographic slices of Fe K (blue), Mn K (green), and Zn K (red) fluorescence lines collected from wildtype Col-0 and vit1-1 seeds, with metal abundances indicated in $\mathrm{mg} \mathrm{kg}^{-1}$ (smaller images) and composite images of iron, manganese and zinc (larger images). Iron in wild-type seeds is localized to the provascular trands of the hypocotyl, radicle and cotyledons, whereas in vit1-1 seeds, it is absent from these cells and located diffusely in the hypocotyl and radicle and epidermal cells of the cotyledons. Modified with permission from ref. 15. 

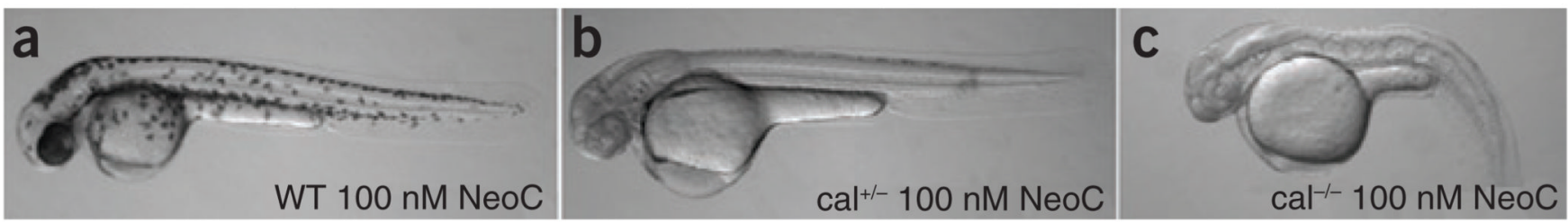

Figure 2.

Gene dosage of atp7a reveals a developmental hierarchy of copper metabolism. (a-c) The copper chelator neocuproine at $100 \mathrm{nM}$ is without effect on melanin pigmentation in wildtype embryos (a), whereas this same dose prevents melanin pigment in atp 7 a (calamity) heterozygotes (b) and worsens the abnormal brain and notochord phenotype in atp7a homozygotes (c). Modified with permission from ref. 26. 